\title{
Clinical significance of herpes virus entry mediator expression in hepatitis B virus-related hepatocellular carcinoma
}

\author{
YONG YI $^{1 *}$, XIAO-CHUN NI ${ }^{2 *}$, GAO LIU $^{1 *}$, YI-RUI YIN ${ }^{1}$, JING-LONG HUANG $^{1}$, WEI GAN ${ }^{1}$, \\ PEI-YUN ZHOU ${ }^{1}$, RUO-YU GUAN ${ }^{1}$, CHENG ZHOU ${ }^{1}$, BAO-YE SUN ${ }^{1}$ and SHUANG-JIAN QIU ${ }^{1}$ \\ ${ }^{1}$ Key Laboratory of Carcinogenesis and Cancer Invasion, Department of Liver Surgery and Transplantation, \\ Ministry of Education, Liver Cancer Institute, Zhongshan Hospital, Fudan University, Shanghai 200032; \\ ${ }^{2}$ Department of General Surgery, Shanghai Ninth People's Hospital of Shanghai JiaoTong University \\ School of Medicine, Shanghai 200000, P.R. China
}

Received November 4, 2019; Accepted April 27, 2020

DOI: $10.3892 / \mathrm{ol} .2020 .11880$

\begin{abstract}
Herpes virus entry mediator (HVEM) is overexpressed in several malignancies, including hepatocellular carcinoma (HCC). However, to the best of our knowledge, the clinical significance of HVEM in hepatitis B virus (HBV)-related HCC remains unclear. Thus, the present study aimed to explore the clinical significance of HVEM in HBV-related HCC. In the present study, HVEM expression was evaluated in HCC cell lines and HCC frozen samples. The prognostic value of HVEM was assessed in a cohort of 221 patients with HBV-related HCC, following radical resection. B- and T-lymphocyte attenuator (BTLA) expression in subsets of $\mathrm{CD}^{+} \mathrm{T}$ cells was determined via flow cytometry analysis. The results demonstrated high HVEM expression in HCC cell lines, and in HCC tissues compared with paired non-cancerous liver tissues. HVEM expression was demonstrated to be significantly associated with tumor encapsulation
\end{abstract}

Correspondence to: Dr Shuang-Jian Qiu, Key Laboratory of Carcinogenesis and Cancer Invasion, Department of Liver Surgery and Transplantation, Ministry of Education, Liver Cancer Institute, Zhongshan Hospital, Fudan University, 180 Fenglin Road, Shanghai 200032, P.R. China

E-mail: qiu.shuangjian@zs-hospital.sh.cn

Contributed equally

Abbreviations: BTLA, B- and T-lymphocyte attenuator; CRC, colorectal cancer; DOI, density of interest; ESCC, esophageal squamous cell carcinoma; HBV, hepatitis B virus; HCC, hepatocellular carcinoma; HCV, hepatitis C virus; HVEM, herpes virus entry mediator; OS, overall survival; PBMCs, peripheral blood mononuclear cells; PILs, peritumor-infiltrating lymphocytes; TILs, tumor-infiltrating lymphocytes; TNF, tumor necrosis factor; Tregs, regulatory T cells; TTR, time to recurrence

Key words: herpes virus entry mediator, B- and T-lymphocyte attenuator, hepatocellular carcinoma, prognosis, immunohistochemistry and vascular invasion. Furthermore, tumor HVEM status was significantly associated with infiltration of regulatory $\mathrm{T}$ cells, but not with $\mathrm{CD}^{+} \mathrm{T}$ cells. Notably, high HVEM expression in $\mathrm{HCC}$ was determined to be an independent predictor of an unfavorable outcome of patients with HCC following radical resection. Higher BTLA expression (the receptor of HVEM) was observed in both HCC-infiltrating $\mathrm{CD}^{+}$effector memory (CCR7- CD45RA') and CD45RA ${ }^{+}$effector memory $\left(\mathrm{CCR}^{-} \mathrm{CD}^{-} \mathrm{RA}^{+}\right.$) T cells in HCC tissues and blood compared with those in paired peritumor tissues or peripheral blood. Taken together, the results of the present study suggest that HVEM may serve a critical role in HBV-related HCC, most likely by promoting tumor progression and tumor immune evasion, thus the HVEM/BLTA signaling pathway may be a potential target in tumor immunotherapy.

\section{Introduction}

Liver cancer was the fourth leading cause of cancer-associated and the sixth most commonly diagnosed cancer worldwide in 2018 (1). Surgical resection, ablation and liver transplantation are the current curative treatment modalities for patients with HCC. However, a high rate of local recurrence or distant metastasis has impeded the improvement of patient outcome following curative treatments (2). Other treatments, such as transcatheter arterial chemoembolization and multi-tyrosine kinase inhibitor are used for advanced HCC (2). Recently, therapeutic monoclonal antibodies targeting immune checkpoint inhibitors (ICIs), such as the programmed cell death protein 1 (PD-1)/programmed death-ligand 1 (PD-L1) axis, have been approved in HCC treatment, and unprecedented improvement in tumor control has been reported (3). However, only a small subset of patients with HCC exhibit a marked response to a single antibody against ICIs, possibly due to the high complexity of the tumor microenvironment (4).

Herpes virus entry mediator (HVEM), a member of the tumor necrosis factor (TNF) receptor super family, interacts with B- and T-lymphocyte attenuator (BTLA), which activates its cytoplasmic domain that contains an inhibitory tyrosine-based motif, attenuating proliferation signals in antigen-activated lymphocytes (5). HVEM is widely expressed 
on multiple cells, such as T, B, natural killer, dendritic and myeloid cells (6). Furthermore, the lung, liver and kidneys have been reported to express HVEM (7). In addition to BTLA, HVEM is also a ligand for CD160, and the TNF superfamily members LIGHT and lymphotoxin- $\beta$ (6). HVEM interacts with CD160 or BTLA to mediate inhibitory signals in T cell proliferation and cytokine secretion (8). Conversely, ligation of HVEM with LIGHT mediates the activation of naïve $T$ cells and clonal expansion (9). HVEM exhibits a bidirectional effect on $\mathrm{T}$ cell activation depending on the engaged ligands. However, the overall function of HVEM is inhibitory, based on the evidence that $\mathrm{HVEM}^{-/-} \mathrm{T}$ cells exhibit an enhanced activation profile and increased susceptibility to the development of Con A mitogen-induced, T cell-dependent autoimmune hepatitis and experimental autoimmune encephalopathy in HVEM-deficient mice (10). Ectopic HVEM expression has been demonstrated to be associated with obesity, autoimmune disease and inflammation $(11,12)$. Furthermore, different types of tumor, including esophageal squamous cell carcinoma (ESCC), HCC and melanoma, exhibit higher HVEM expression in tumors compared with adjacent normal tissues (13-15). Downregulation of HVEM in ESCC cells induces cell cycle arrest and inhibits tumor growth in vivo (13). An inverse association between HVEM and tumor-infiltrating immune cells, including $\mathrm{CD}^{+}, \mathrm{CD}^{+}$and $\mathrm{CD} 45 \mathrm{RO}^{+}$lymphocytes has been reported in both human ESCC and hepatitis $\mathrm{C}$ virus (HCV)-related HCC $(13,14)$. Notably, high HVEM expression in cancer serves as an independent predictor of poor survival outcomes in patients with ESCC or HCC, following radical resection $(13,14)$. However, the expression status and clinical significance of HVEM in HCC with hepatitis B virus (HBV) remain largely unknown.

Thus, the present study aimed to investigate the clinical significance of HVEM in HBV-related HCC, and determine the association between HVEM and subsets of HCC-infiltrating immune cells. Furthermore, BTLA expression in subsets of $\mathrm{CD}^{+} \mathrm{T}$ cells was investigated.

\section{Materials and methods}

Patients and specimens. The clinicopathological characteristics of patients with HCC included in the current study are presented in Table SI. For tissue microarray construction, 221 patients with a median age of 52 years and age range, 18-81 years, who underwent radical resection for HCC at the Department of Liver Surgery and Transplantation, Zhongshan Hospital (Shanghai, China) were enrolled between April 2002 and December 2007. The patients were comprised of 188 males and 33 females. The inclusion and exclusion criterion of the cohort are described in previous studies $(16,17)$. The patient cohort was divided into the $\mathrm{HVEM}^{\text {high }}$ group $\left(\geq 50 \%\right.$ of $\mathrm{HVEM}^{+}$ tumor cells; $\mathrm{n}=139)$ and $\mathrm{HVEM}^{\text {low }}$ group $\left(<50 \%\right.$ of $\mathrm{HVEM}^{+}$ tumor cells; $n=82$ ). Follow-up was performed until mortality or May 2017. Patients were followed up every 2 months during the first postoperative year and then every 3 to 4 months for the remainder of the duration. The median follow-up for all patients was 53 months, with a range of 2-180 months. Time to recurrence (TTR) and overall survival (OS) time were calculated as the interval between primary surgical resection and the first recurrence or mortality, respectively. Briefly, the inclusion criteria were as follows: i) Underwent radical resection for HCC with distinctive pathological diagnosis; ii) no preoperative anticancer treatment or extrahepatic metastasis; and iii) complete follow-up data. Paired peripheral blood and fresh tissue samples were obtained from 20 patients with HCC randomly from the total patient cohort for lymphocyte isolation. Frozen HCC tissue samples and adjacent liver tissues $(1 \mathrm{~cm}$ away from tumor tissues) from another 28 patients with HCC were randomly selected from the tissue bank of Zhongshan Hospital for PCR analysis. The present study was approved by the Zhongshan Hospital Research Ethics Committee (approval no. Y2017-186) and written informed consent was provided by all patients prior to the study.

Reverse transcription-quantitative $(R T-q) P C R$. Following radical resection of HCC tissue samples and adjacent liver tissues, RNA extraction was immediately performed, and both tissue samples and extracted RNA were preserved at $-80^{\circ} \mathrm{C}$. Total RNA was extracted from frozen tissue samples using TRIzol reagent (Invitrogen; Thermo Fisher Scientific, Inc.) and reverse transcribed into complementary DNA at $45^{\circ} \mathrm{C}$ (cDNA; $0.5 \mu \mathrm{g}$ ) using the cDNA Reverse Transcription kit (Applied Biosystems; Thermo Fisher Scientific, Inc.). qPCR was subsequently performed using the TaqMan Universal PCR Master Mix (Thermo Fisher Scientific, Inc.) with $1 \mu 1$ cDNA in a $25 \mu \mathrm{l}$ final reaction volume and an ABI Prism 7300 system (Applied Biosystems; Thermo Fisher Scientific, Inc.). The following primer sequences were used for qPCR: HVEM forward, 5'-CTTGAGGCTGGTGCTGTATC-3' and reverse, 5'-GGTGGGCAATGTAGGTG-3'; and GAPDH forward, 5'-CACCCACTCCTCCACCTTTG-3' and reverse, 5'-CCA CCACCCTGTTGCTGTAG-3'. The following thermocycling conditions were used for qPCR: Initial denaturation at $95^{\circ} \mathrm{C}$ for $10 \mathrm{~min}$; 40 cycles of denaturation at $95^{\circ} \mathrm{C}$ for $15 \mathrm{sec}$, annealing at $55^{\circ} \mathrm{C}$ for $45 \mathrm{sec}$ and extension at $60^{\circ} \mathrm{C}$ for $15 \mathrm{sec}$. Relative HVEM expression levels were calculated using the $2^{-\Delta \Delta \mathrm{Cq}}$ method (18) and normalized to the internal reference gene GAPDH.

Immunohistochemistry (IHC) and evaluation. Tissue microarray was constructed as previously described $(16,17)$. The tissues were fixed by immersion in a $10 \%$ formalin solution for $4-8 \mathrm{~h}$ at room temperature. The thickness of sections was 5-15 $\mu \mathrm{m}$. Briefly, following deparaffinization and rehydration in a graded series of ethanol (100, 95, 80 and 50\%), sections were incubated with $0.3 \% \mathrm{H}_{2} \mathrm{O}_{2}$ for $20 \mathrm{~min}$ at room temperature to inhibit endogenous peroxidase activity. Antigen retrieval was performed with Tris-ETDA ( $\mathrm{pH}$ 9.0) using a microwave oven at $99-100^{\circ} \mathrm{C}$ for $20 \mathrm{~min}$. Subsequently, sections were blocked with 5\% BSA (Sigma-Aldrich; Merck KGaA) at room temperature for $30 \mathrm{~min}$, prior to incubation with primary antibodies directed against human HVEM/TNFRSF14 (ab47677; 1:20; Abcam), human CD8 (1:50; Dako; Agilent Technologies, Inc.) and human forkhead box P3 (FOXP3; cat. no. ab20034; 1:100; Abcam) overnight at $4^{\circ} \mathrm{C}$. A $100 \mu \mathrm{l}$ of diluted biotinylated secondary antibody (1:1,000; cat. no. ab98624; Abcam) was then added and the sections were incubated in a humidified chamber at room temperature for $30 \mathrm{~min}$. The components of the Histostain ${ }^{\circledR}$-Plus kit (Invitrogen; Thermo Fisher Scientific, Inc.) were used for signal amplification and visualization. 
Immunohistochemical staining for HVEM was blindly evaluated by two independent pathologists using confocal laser-scanning microscope (magnification, x100) (Olympus FluoView FV1000; Olympus Corporation), as previously described $(13,15)$. Tumor cells $(\geq 1,000)$ were counted and the percentage of tumor cells with positive staining was calculated. Integrated absorbance and the area in a photograph was measured using Image-Pro Plus software (version 6.0; Media Cybernetic, Inc.), in order to compare the expression levels of HVEM between HCC and peritumor tissue samples. The density of interest (DOI) was calculated as the product of integrated absorbance/total area. Tumor-infiltrating $\mathrm{CD}^{+}$ and $\mathrm{FOXP}^{+} \mathrm{T}$ cells were counted in five randomly selected fields (magnification, $\mathrm{x} 400$ ) per sample by two independent investigators.

Cell lines. Human HCC cell lines, including HCCLM3, MHCC97H and MHCC97L [human HCC cell lines with high, moderate and low metastatic potential, respectively, which were derived from the same parental cell line and established in Liver Cancer Institute, Zhongshan Hospital, Fudan University (19,20)], and PLC (Japanese Cancer Research Bank) were used in the present study. All cell lines were maintained in DMEM (Thermo Fisher Scientific, Inc.) containing 10\% FBS (Thermo Fisher Scientific, Inc.) supplemented with $100 \mathrm{IU} / \mathrm{ml}$ penicillin and $100 \mu \mathrm{g} / \mathrm{ml}$ streptomycin and incubated at $37^{\circ} \mathrm{C}$ with $5 \% \mathrm{CO}_{2}$.

Immunocytochemistry. Immunocytochemistry was performed according to standardized protocols (21). Briefly, seed adheren $\mathrm{T}$ cells were cultured in 6 -well tissue plates in a sterile tissue culture hood at room temperature overnight and then fixed with $4 \%$ formaldehyde for $10 \mathrm{~min}$ at room temperature and permeabilized using $0.2 \%$ Triton X-100 at room temperature for $15 \mathrm{~min}$. Subsequently, cells were blocked with $1 \%$ BSA for $30 \mathrm{~min}$ at room temperature, prior to incubation with anti-human HVEM/TNFRSF14 antibody (1:10; cat. no. ab47677; Abcam) overnight at $4^{\circ} \mathrm{C}$. Next, the cells were incubated with the secondary antibody $(1: 2,000$; cat. no. ab205718; Abcam) for $1 \mathrm{~h}$ at room temperature. The components of the Alexa Fluor Plus 488 (Invitrogen; Thermo Fisher Scientific, Inc.) were used for signal amplification, and DAPI at $300 \mathrm{ng} / \mathrm{ml}$ incubated for $10 \mathrm{~min}$ away from light was used as a nuclear counterstain. The slides were observed under a confocal laser-scanning microscope (magnification, x100) (Olympus FluoView FV1000; Olympus Corporation).

Cell isolation and flow cytometric analysis. Isolation of peripheral blood mononuclear cells (PBMCs), peritumor-infiltrating lymphocytes (PILs) and tumor-infiltrating lymphocytes (TILs) was performed as previously described (16). Briefly, PBMCs were isolated by Ficoll density gradient centrifugation, according to the manufacturer's protocol (GE Healthcare). PILs and TILs were isolated from clinical HCC specimens by Percoll gradient centrifugation as previously described (22). Analysis of surface antigen expression was performed, according to the manufacturer's protocol (BD Biosciences). Briefly, BTLA expression on $\mathrm{CD}^{+} \mathrm{T}$ cells was investigated in relation to the differentiation stage discriminated by the expression of chemokine receptor CCR7 in combination with the naïve cell marker CD45RA. $\mathrm{CD}^{+} \mathrm{T}$ cells were gated as follows: i) Naïve $\left(\mathrm{CCR}^{+} \mathrm{CD}^{2} 5 \mathrm{RA}^{+}\right)$; ii) central memory (TCM, CCR7 ${ }^{+} \mathrm{CD} 45 \mathrm{RA}^{-}$); iii) effector memory (CCR7CD45RA'); and iv) effector memory RA (CCR7- ${ }^{-}$CD45RA $^{+}$). $1 \times 10^{6}$ PBMCs, PILs and TILs were washed in PBS supplemented with $1 \%$ BSA and $0.1 \%$ sodium azide (Sigma-Aldrich; Merck KGaA) three times, and incubated with anti-BTLA (1:10; cat. no. 746759, BD Pharmingen) for $30 \mathrm{~min}$ at $4^{\circ} \mathrm{C}$. Following washing three times using ice cold PBS, $10 \%$ FCS, $1 \%$ sodium azide and centrifugation at $400 \mathrm{x}$ g at $4^{\circ} \mathrm{C}$ for $5 \mathrm{~min}$, samples were fixed in $\mathrm{PBS} / 1 \%$ paraformaldehyde (Sigma-Aldrich; Merck KGaA) overnight at $4^{\circ} \mathrm{C}$. Lymphocytes were gated based on forward and side scatters, and at least $1 \times 10^{5}$ gated events were acquired for each sample and analyzed using FlowJo v.10.5.3 software (FlowJo LLC).

Statistical analysis. Statistical analysis was performed using SPSS software (version 16.0; SPSS, Inc.). Paired Student's $\mathrm{t}$-tests were used for comparing two groups. A $\chi^{2}$ test was used to determine the association between HVEM expression and clinicopathological characteristics of patients with HCC. One-way ANOVA followed by Bonferroni's post-hoc test was used to compare differences between multiple groups. Univariate survival analysis was performed using the Kaplan-Meier method and the log-rank test, while Cox multivariate analysis was performed to adjust for potential confounding variables in a stepwise manner (forward, likelihood ratio) and determine the independent prognostic factors. Data are shown as the mean \pm standard deviation of three independent experiments, and t-tests were used to compare group averages. $\mathrm{P}<0.05$ was considered to indicate a statistically significant difference.

\section{Results}

HVEM expression in HBV-related HCC and HCC cell lines. RT-qPCR was performed to determine HVEM expression in patients with HCC with a background of HBV. The results demonstrated that HVEM expression was significantly higher in HCC tissues compared with paired peritumor tissues $(\mathrm{P}=0.0066$; Fig. 1A). Furthermore, HVEM expression was assessed in HCC via IHC analysis. Positive staining of HVEM was identified predominantly on the membrane and in the cytoplasm of tumor cells. Scatter positive staining of the stromal cells was also observed in peritumor tissue, and accidentally observed embolus also demonstrated higher HVEM expression compared with surrounding liver tissue (Fig. 1B). HCC tissue was demonstrated to have a significantly stronger HVEM expression intensity when evaluated by DOI (0.035 \pm 0.021 vs. $0008 \pm 0.009$; P<0.0001; Fig. 1C). Furthermore, high HVEM expression was demonstrated in HCC cell lines via immunocytochemistry analysis (Fig. 1D).

Association between HVEM expression and clinicopathological characteristics of HCC. The patient cohort was divided into the $\mathrm{HVEM}^{\text {high }}$ group ( $\geq 50 \%$ of $\mathrm{HVEM}^{+}$tumor cells, $\mathrm{n}=139)$ and $\mathrm{HVEM}^{\text {low }}$ group $\left(<50 \%\right.$ of $\mathrm{HVEM}^{+}$tumor cells, $n=82)$, as previously described $(10,11)$. Vascular invasion was significantly higher and tumor encapsulation was significantly lower in the HVEM ${ }^{\text {high }}$ group compared with 
A

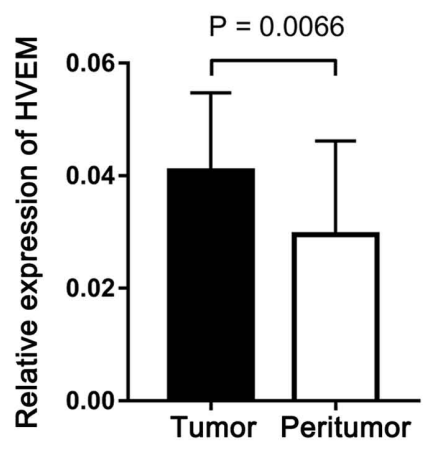

C

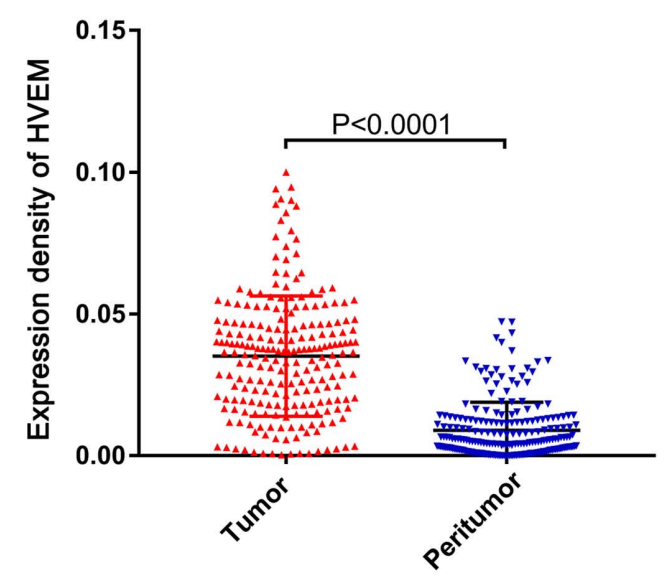

B

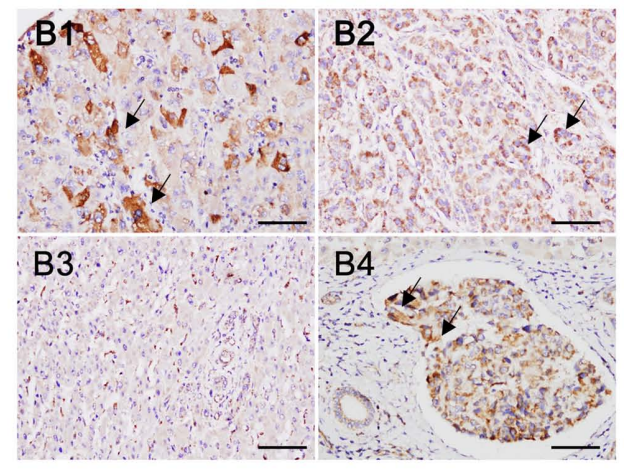

HVEM

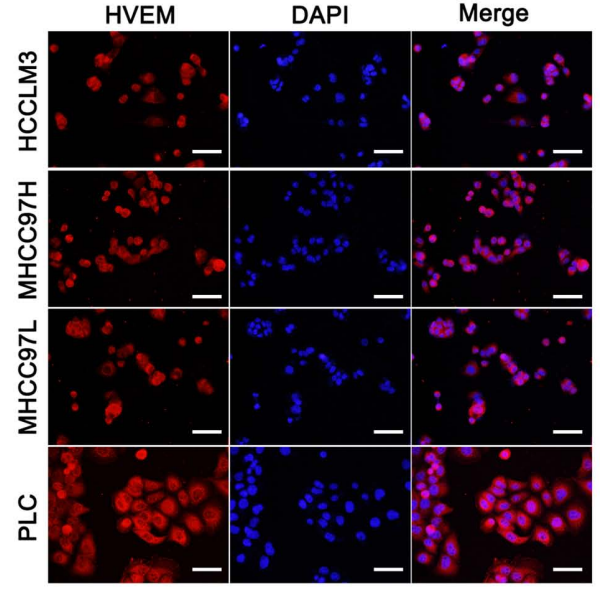

Figure 1. HVEM expression in surgically resected HCC tissues. (A) Reverse transcription-quantitative PCR analysis demonstrated that HVEM expression was significantly higher in HCC tissues compared with peritumoral liver tissues $(\mathrm{n}=20)$. (B) Representative images of HVEM expression in HCC. (B-1 and B-2) Positive staining of HVEM was predominantly exhibited on the cytomembrane and in the cytoplasm of tumor cells and is indicated by the black arrows. (B-3) Peritumor tissues demonstrated relatively low HVEM expression. (B-4) High HVEM expression in tumor embolus was observed and is indicated by the black arrows. Scale bar, $50 \mu \mathrm{m}$. (C) Density of HVEM staining was evaluated by density of interest (mean \pm standard deviation). (D) Immunocytochemical staining of HVEM in HCC cell lines. DAPI was used as a nuclear counterstain. Scale bar, $50 \mu \mathrm{m}$. HVEM, herpes virus entry mediator; HCC, hepatocellular carcinoma.

the HVEM ${ }^{\text {low }}$ group $(\mathrm{P}=0.048$ and $\mathrm{P}=0.036$, respectively; Table I). Furthermore, tumors with high HVEM expression had a relatively higher rate of advanced BCLC stage; however, no significant difference was observed between the groups $(\mathrm{P}=0.087$; Table I). AFP level and TNM stage failed to demonstrate a prognostic value for patients with $\mathrm{HCC}(\mathrm{P}=0.081$ and $\mathrm{P}=0.211$, respectively; Table I). Taken together, these results suggest that HVEM may be involved in disease progression of patients with HCC.

Association between HVEM and $\mathrm{FOXP}^{+} \mathrm{T}$ cells in patients with HCC. HVEM may be involved in tumor progression by inducing the immune escape of tumor cells (23). The present study evaluated the associations between HVEM with $\mathrm{CD}^{+} \mathrm{T}$ cells and $\mathrm{FOXP}^{+}$regulatory $\mathrm{T}$ cells (Tregs). High FOXP3 expression was significantly higher in the HVEM ${ }^{\text {high }}$ group compared with the HVEM $^{\text {low }}$ group $(\mathrm{P}=0.005)$; however, no significant association was observed between HVEM expression and $\mathrm{CD}^{+} \mathrm{T}$ cells (Table I).

Prognostic significance of HVEM in HBV-related $H C C$. Kaplan-Meier analysis demonstrated that high HVEM expression in HCC tissue was associated with a shorter TTR time and shorter OS time compared with the HVEM ${ }^{\text {low }}$ group $(\mathrm{P}<0.001$; Fig. 2). Factors that demonstrated significance by univariate analysis presented in Table II were enrolled in a multivariate Cox proportional hazards model. We found that $\alpha$-fetoprotein, $\gamma$-glutamyl transferase, tumor size, vascular invasion, FOXP3 and CD8 are independent prognostic factor for predicting OS. Importantly, HVEM was revealed to be an independent prognostic factor for predicting recurrence and OS (Table II).

BTLA expression in $C D 8^{+} T$ cells. $\mathrm{CD}^{+} \mathrm{T}$ cells act as the main effector cells in the tumor microenvironment (24). As expected, the infiltration of $\mathrm{CD}^{+} \mathrm{T}$ cells decreased in $\mathrm{HCC}$ compared with that in paired peritumor tissue and peripheral blood (Fig. 3A). The ligation of BTLA by HVEM expressed by HCC cells may result in decreased T cell proliferation and cytokine secretion (25). Thus, BTLA expression on $\mathrm{CD}^{+} \mathrm{T}$ cells was investigated in relation to the differentiation stage discriminated by the expression of chemokine receptor CCR7 in combination with the naïve cell marker CD45RA. CD8 ${ }^{+}$ $\mathrm{T}$ cells were gated as follows: i) Naïve $\left(\mathrm{CCR} 7^{+} \mathrm{CD} 45 \mathrm{RA}^{+}\right)$; ii) central memory (TCM, CCR7 $\left.{ }^{+} \mathrm{CD} 45 \mathrm{RA}{ }^{-}\right)$; iii) effector memory (CCR7- CD45RA') and iv) effector memory RA 
Table I. Association between HVEM expression and clinicopathological characteristics of patients with hepatocellular carcinoma $(n=221)$.

\begin{tabular}{|c|c|c|c|c|}
\hline \multirow[b]{2}{*}{ Characteristic } & \multirow[b]{2}{*}{ Number of patients, $\mathrm{n}$} & \multicolumn{3}{|c|}{ HVEM expression } \\
\hline & & Low, n & High, $n$ & P-value \\
\hline Age, years & & & & $>0.999$ \\
\hline$\leq 52$ & 110 & 69 & 41 & \\
\hline$>52$ & 111 & 70 & 41 & \\
\hline Sex & & & & 0.050 \\
\hline Male & 188 & 113 & 75 & \\
\hline Female & 33 & 26 & 7 & \\
\hline HBV history & & & & 0.606 \\
\hline Yes & 204 & 127 & 77 & \\
\hline No & 17 & 12 & 5 & \\
\hline Liver cirrhosis & & & & 0.534 \\
\hline Yes & 193 & 123 & 70 & \\
\hline No & 28 & 16 & 12 & \\
\hline AFP, ng/ml & & & & 0.081 \\
\hline$\leq 20$ & 79 & 56 & 23 & \\
\hline$>20$ & 142 & 83 & 59 & \\
\hline$\gamma$-GT, U/l & & & & 0.576 \\
\hline$\leq 56$ & 98 & 64 & 34 & \\
\hline$>56$ & 123 & 75 & 48 & \\
\hline Tumor size, $\mathrm{cm}$ & & & & 0.051 \\
\hline$\leq 5$ & 114 & 79 & 35 & \\
\hline$>5$ & 107 & 60 & 47 & \\
\hline Tumor number & & & & $>0.999$ \\
\hline Single & 172 & 108 & 64 & \\
\hline Multiple & 49 & 31 & 18 & \\
\hline Tumor encapsulation & & & & 0.036 \\
\hline None & 113 & 79 & 34 & \\
\hline Complete & 108 & 60 & 48 & \\
\hline Tumor differentiation & & & & 0.205 \\
\hline I-II & 131 & 87 & 44 & \\
\hline III-IV & 90 & 52 & 38 & \\
\hline Vascular invasion & & & & $0.048^{\mathrm{a}}$ \\
\hline Yes & 130 & 89 & 41 & \\
\hline No & 91 & 50 & 41 & \\
\hline TNM stage & & & & 0.211 \\
\hline I & 107 & 72 & 35 & \\
\hline II-III & 114 & 67 & 47 & \\
\hline BCLC stage & & & & 0.087 \\
\hline A & 80 & 57 & 23 & \\
\hline $\mathrm{B}$ & 50 & 32 & 18 & \\
\hline $\mathrm{C}$ & 91 & 50 & 41 & \\
\hline FOXP3 & & & & 0.005 \\
\hline Low & 117 & 84 & 33 & \\
\hline High & 104 & 55 & 49 & \\
\hline CD8 & & & & 0.676 \\
\hline Low & 108 & 66 & 42 & \\
\hline High & 113 & 73 & 40 & \\
\hline
\end{tabular}

HVEM, herpes virus entry mediator; HBV, hepatitis B virus; AFP, $\alpha$-fetoprotein; $\gamma$-GT, $\gamma$-glutamyl transferase; TNM, tumor-node-metastasis; BCLC, Barcelona clinic liver cancer; FOXP3, forkhead box protein 3. TNM stage, BCLC stage and tumor differentiation were based on previous studies (39-41). 
Table II. Univariate and multivariate analyses of prognostic factors in patients with hepatocellular carcinoma ( $\mathrm{n}=221)$.

\begin{tabular}{|c|c|c|c|c|c|c|}
\hline \multirow[b]{3}{*}{ Factor } & \multicolumn{3}{|c|}{ Overall survival time } & \multicolumn{3}{|c|}{ Time to recurrence } \\
\hline & \multirow{2}{*}{$\begin{array}{c}\text { Univariate } \\
\text { P-value }\end{array}$} & \multicolumn{2}{|c|}{ Multivariate analysis } & \multirow{2}{*}{$\begin{array}{l}\text { Univariate } \\
\text { P-value }\end{array}$} & \multicolumn{2}{|c|}{ Multivariate analysis } \\
\hline & & $\mathrm{HR}(95 \% \mathrm{CI})$ & P-value & & $\mathrm{HR}(95 \% \mathrm{CI})$ & P-value \\
\hline$\alpha$-fetoprotein, $\mathrm{ng} / \mathrm{ml}$ ( $\leq 20$ vs. $>20$ ) & 0.001 & $1.553(1.054-2.288)$ & 0.026 & 0.066 & NA & NA \\
\hline$\gamma$-glutamyl transferase, units/l ( $\leq 56$ vs. $>56$ ) & $<0.001$ & $1.845(1.297-2.625)$ & 0.001 & 0.089 & NA & NA \\
\hline Liver cirrhosis (No vs. Yes) & 0.033 & NS & NS & 0.076 & NA & NA \\
\hline Tumor size, $\mathrm{cm}(\leq 5$ vs. $>5$ ) & $<0.001$ & $1.436(1.006-2.050)$ & 0.046 & 0.002 & NS & NS \\
\hline Tumor multiplicity (Single vs. Multiple) & 0.001 & NS & NS & 0.001 & $1.785(1.217-2.617)$ & 0.003 \\
\hline Tumor encapsulation (Complete vs. None) & 0.009 & NS & NS & 0.003 & NS & NS \\
\hline Tumor differentiation (I/II vs. III/IV) & 0.082 & NA & NA & 0.248 & NA & NA \\
\hline Vascular invasion(No vs. Yes) & $<0.001$ & $2.974(2.057-4.298)$ & $<0.001$ & $<0.001$ & $2.895(2.037-4.117)$ & $<0.001$ \\
\hline HVEM (Positive vs. Negative) & $<0.001$ & $2.162(1.528-3.059)$ & $<0.001$ & 0.003 & $1.752(1.222-2.513)$ & 0.002 \\
\hline FOXP3 (High vs. Low) & $<0.001$ & $2.314(1.630-3.284)$ & $<0.001$ & 0.013 & NS & NS \\
\hline CD8 (High vs. Low) & 0.001 & $0.523(0.367-0.746)$ & $<0.001$ & 0.084 & NA & NA \\
\hline
\end{tabular}

The Kaplan-Meier method and log-rank test was used for univariate analysis, while the Cox multivariate proportional hazards regression model, with a stepwise manner (forward, likelihood ratio) was adopted for subsequent analysis. HR, hazard ratio; CI, confidence interval; HVEM, herpes virus entry mediator; FOXP3, forkhead box protein 3; NS, not significant; NA, not applicable.

A

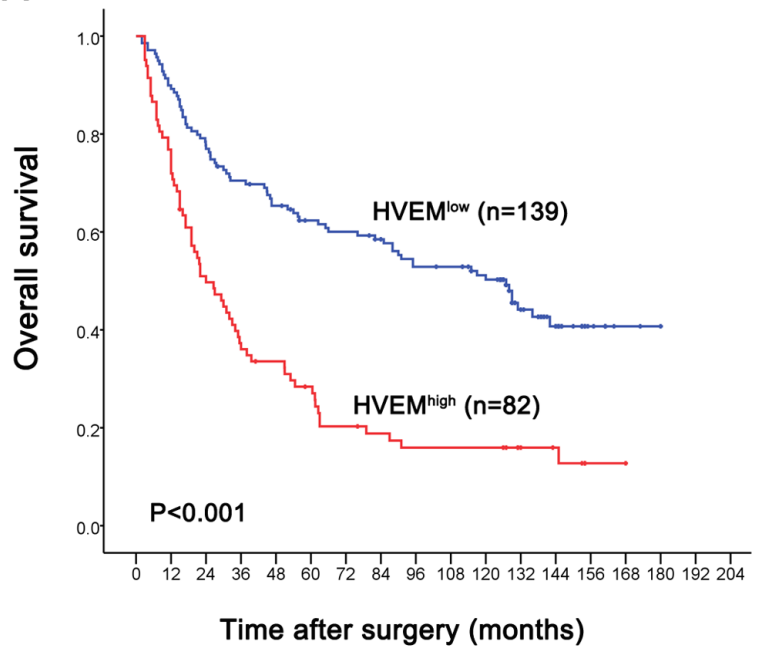

B

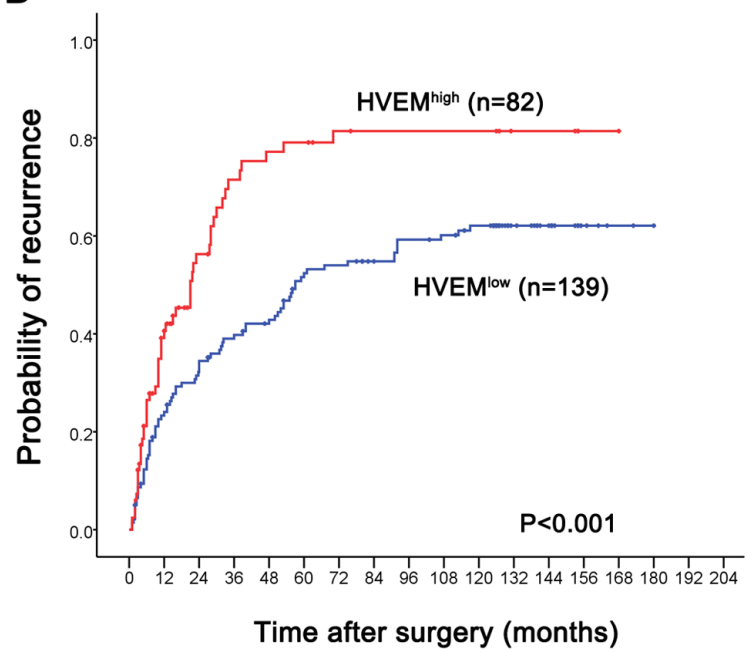

Figure 2. Clinical significance of HVEM in patients with hepatocellular carcinoma following radial resection. (A) The HVEM ${ }^{\text {high }}$ group had a shorter overall survival time compared with the $\mathrm{HVEM}^{\text {low }}$ group. (B) The probability of recurrence was higher in the $\mathrm{HVEM}^{\text {high }}$ group compared with the HVEM ${ }^{\text {low }}$ group. $\mathrm{P}<0.001$. HVEM, herpes virus entry mediator.

$\left(\mathrm{CCR}^{-} \mathrm{CD}^{-} 5 \mathrm{RA}^{+}\right)$. The results demonstrated that the surface expression of BTLA in these cell subtypes gradually decreased with differentiation stage (Fig. 3A). However, HCC-infiltrating $\mathrm{CD}^{+} \mathrm{T}$ cells still exhibited persistently high levels of BTLA. Specifically, both effector memory and effector memory RA $\mathrm{CD}^{+} \mathrm{T}$ cells exhibited higher levels of BTLA in HCC tissues compared with peritumor tissues (Fig. 3B).

\section{Discussion}

It has been demonstrated that HVEM plays dynamic immune regulatory functions in various physiological and pathological conditions $(6,12)$. In the tumor microenvironment, HVEM is involved in tumor immune evasion through ligation with BTLA, a coinhibitory receptor with functional similarities to PD-1 and CTLA-4 (3). The present study demonstrated a significantly higher expression of HVEM in HCC compared with paired peritumor tissue. Furthermore, high HVEM expression was associated with poor clinical outcome and invasive characteristics, such as high rate of vascular invasion and infiltration of suppressive Tregs. It has been reported that $\mathrm{HCC}$-infiltrating $\mathrm{CD}^{+} \mathrm{T}$ cells differentiate from naïve to effector cells, and these cells were important in HCC and were demonstrated to persistently express high levels of BTLA (26), 

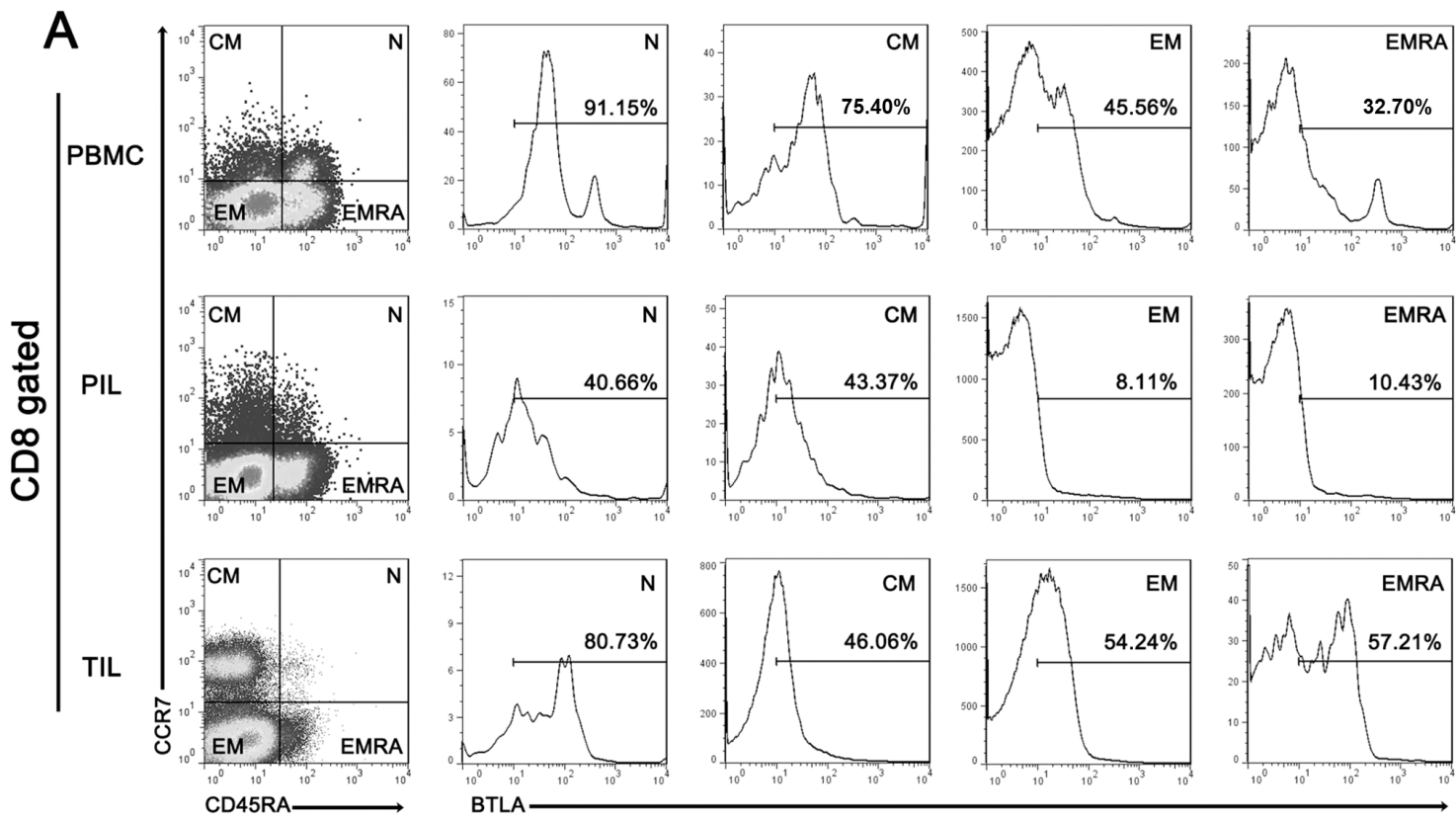

B

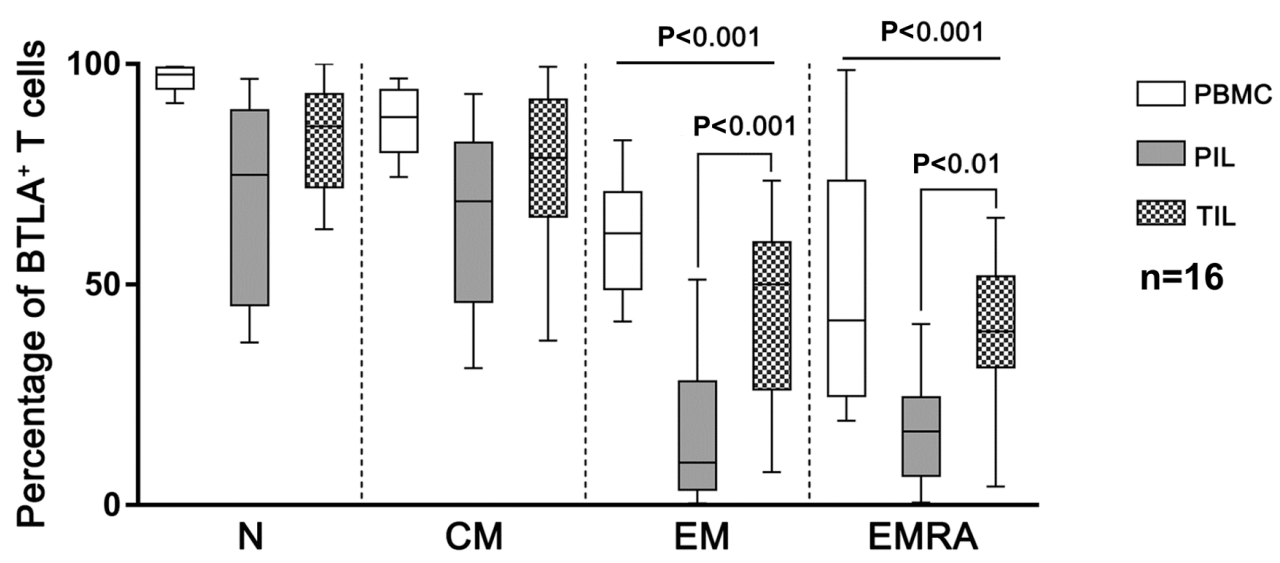

Figure 3. BTLA expression in HCC-infiltrating $\mathrm{CD} 8^{+} \mathrm{T}$ cells. (A) Representative figures of BTLA expression in CD8 ${ }^{+} \mathrm{T}$ cells from peripheral blood, peritumor tissue and tumor tissue of patients with $\mathrm{HCC}$. CD8 ${ }^{+} \mathrm{T}$ cells were divided into four subsets according to CD45RA and CCR7 expression $\left(\mathrm{N}, \mathrm{CCR} 7^{+} \mathrm{CD} 45 \mathrm{RA} \mathrm{A}^{+}\right.$

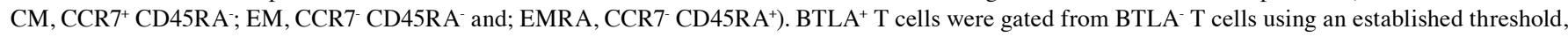
according to the autologous naïve $\mathrm{T}$ cell subsets of PBMC, which is always BTLA positive. (B) Statistical analysis of BTLA ${ }^{+} \mathrm{CD} 8^{+} \mathrm{T}$ cell subsets in PBMCs, PILs and TILs derived from patients with HCC. HCC, hepatocellular carcinoma; CD8, cluster of differentiation 8; BTLA, B- and T-lymphocyte attenuator; PBMCs, peripheral blood mononuclear cells; PILs, peritumor-infiltrating lymphocytes; TILs, tumor-infiltrating lymphocytes; N, naïve; CM, central memory, EM, effector memory; EMRA, effector memory RA ${ }^{+}$.

which underlines the importance of the HVEM/BTLA signaling pathway in HCC.

Immunotherapy based on ICIs has reported promising therapeutic outcomes in patients with cancer (27). PD-1/PD-L1 and CTLA-4 inhibitors have been approved for certain cancer treatments, and some are currently under clinical trials (4). However, the low response rate is one of the major difficulties for ICI-based treatments in some patients with cancer (28). In HCC, it has been reported that only $10-30 \%$ of treated patients respond to anti-PD-1/PD-L1 therapy (29). Conversely, several other ICIs, including HVEM/BTLA, CD73 and mucin domain 3 , also regulate immune responses in tumor niches and may be alternative targets for novel immune therapy (3). The
HVEM/BTLA signaling pathway is considered a novel target for checkpoint blockade, based on the fact that HVEM/BTLA inhibition enhances human $\mathrm{T}$ cell responses when used alone or in combination with anti-PD-1 treatment (30-33). It is reasonable to expect the combination of HVEM blockade and other anticancer treatments, such as resection, ablation, chemotherapy and anti-PD-1/PD-L1 treatment, may induce a synergistic anticancer effect. However, clinical trials are required to effectively evaluate the combined modality.

HVEM is involved in cancer progression through mediating immune evasion; a higher expression of HVEM in cancer tissue is associated with relatively poorer survival outcomes, as reported in patients with ESCC, 
HCV-related hepatocellular carcinoma and colorectal cancer (CRC) $(13,14,34)$. However, to the best of our knowledge, the significance of HVEM in HBV-related HCC was previously undetermined. The present study verified the prognostic role of HVEM in HBV-related HCC. Furthermore, a significant association between HVEM and aggressive biological behavior of HCC, including vascular invasion and incomplete tumor capsule, was identified. Similarly, overexpression of HVEM in patients with non-small cell lung carcinoma of N2 lymph node metastasis or late-stage has been observed (24). In CRC and gastric cancer, HVEM status is significantly associated with tumor status and pathological stage $(34,35)$. Conversely, the present study determined that HVEM expression levels are associated with tumor-infiltrating Tregs, a robust immune inhibitor, rather than $\mathrm{CD}^{+} \mathrm{T}$ cells (36). Notably, Tao et al (36) reported that Tregs exert their suppressive effect via the upregulation of HVEM, which, upon ligation with BTLA expressed on effector cells, helps control immune response. $\mathrm{HVEM}^{-/}$ Tregs have been demonstrated to decrease suppressive activity compared with wild-type Tregs (36). These findings suggest that immunotherapy targeting HVEM may lead to activation of effector T cells and dampening of Tregs.

Studies have reported that HVEM can activate BTLA, thus inhibiting $\mathrm{CD}^{+} \mathrm{T}$ cell differentiation and cytokine secretion $(37,38)$. The present study identified aberrant persistent high expression of BTLA by differentiated effector T cells derived from HCC tissue, suggesting the HVEM/BTLA signaling pathway may play a role in the inhibition of efficient immune responses against cancer.

Overall, the results of the present study suggest a prognostic value of HVEM in patients with HCC following radical resection. Thus, the HVEM/BTLA signaling pathway may be a target in cancer immunotherapy.

\section{Acknowledgements}

Not applicable.

\section{Funding}

The present study was funded by the National Key Research and Development Program of China (grant nos. 2017YFC0908101 and 2017YFC0908102), the National Natural Science Foundation of China (grant no. 81772510), Research Programs of Science and Technology Commission Foundation of Shanghai (grant nos. 16DZ0500300 and 18XD1401100), the Developing Foundation of Zhongshan (grant no. 2019ZSFZ24) and Shanghai Municipal Key Clinical Specialty.

\section{Availability of data and materials}

All data generated or analyzed during the present study are included in this published article.

\section{Authors' contributions}

YY and SJQ designed the present study and contributed to the development of methodology. YY and JLH contributed to the acquisition of data. XCN and GL performed the experiments. JLH, WG, PYZ, RYG, CZ, YRY and BYS analyzed the data and performed the studies. YY drafted the manuscript and reviewed the manuscript for important intellectual content and SJQ acquired the funding. YY contributed to the administrative, technical, or material support. All authors read and approved the final manuscript.

\section{Ethics approval and consent to participate}

The present study was approved by the Zhongshan Hospital Research Ethics Committee (approval no. Y2017-186), and written informed consent was provided by all patients prior to the study start.

\section{Patient consent for publication}

Not applicable.

\section{Competing interests}

The authors declare that they have no competing interests.

\section{References}

1. Bray F, Ferlay J, Soerjomataram I, Siegel RL, Torre LA and Jemal A: Global cancer statistics 2018: GLOBOCAN estimates of incidence and mortality worldwide for 36 cancers in 185 countries. CA Cancer J Clin 68: 394-424, 2018.

2. Villanueva A: Hepatocellular carcinoma. N Engl J Med 380: 1450-1462, 2019.

3. Greten TF and Sangro B: Targets for immunotherapy of liver cancer. J Hepatol: Sep 18, 2017 (Epub ahead of print).

4. Okusaka T and Ikeda M: Immunotherapy for hepatocellular carcinoma: Current status and future perspectives. ESMO Open 3 (Suppl 1): e000455, 2018

5. Cheung TC, Humphreys IR, Potter KG, Norris PS, Shumway HM, Tran BR, Patterson G, Jean-Jacques R, Yoon M, Spear PG, et al: Evolutionarily divergent herpesviruses modulate $\mathrm{T}$ cell activation by targeting the herpesvirus entry mediator cosignaling pathway. Proc Natl Acad Sci USA 102: 13218-13223, 2005.

6. Cai G and Freeman GJ: The CD160, BTLA, LIGHT/HVEM pathway: A bidirectional switch regulating T-cell activation. Immunol Rev 229: 244-258, 2009.

7. Yu X, Zheng Y, Mao R, Su Z and Zhang J: BTLA/HVEM signaling: Milestones in research and role in chronic hepatitis B virus infection. Front Immunol 10: 617, 2019.

8. Rodriguez-Barbosa JI, Schneider P, Weigert A, Lee KM, Kim TJ, Perez-Simon JA and Del Rio ML: HVEM, a cosignaling molecular switch, and its interactions with BTLA, CD160 and LIGHT. Cell Mol Immunol 16: 679-682, 2019.

9. delRioML,Lucas CL,BuhlerL, RayatGandRodriguez-BarbosaJI: HVEM/LIGHT/BTLA/CD160 cosignaling pathways as targets for immune regulation. J Leukoc Biol 87: 223-235, 2010.

10. Wang Y, Subudhi SK, Anders RA, Lo J, Sun Y, Blink S, Wang Y, Wang J, Liu X, Mink K, et al: The role of herpesvirus entry mediator as a negative regulator of T cell-mediated responses. J Clin Invest 115: 711-717, 2005.

11. Croft M, Duan W, Choi H, Eun SY, Madireddi S and Mehta A: TNF superfamily in inflammatory disease: Translating basic insights. Trends Immunol 33: 144-152, 2012.

12. Shui JW, Steinberg MW and Kronenberg M: Regulation of inflammation, autoimmunity, and infection immunity by HVEM-BTLA signaling. J Leukoc Biol 89: 517-523, 2011.

13. Migita K, Sho M, Shimada K, Yasuda S, Yamato I, Takayama T, Matsumoto S, Wakatsuki K, Hotta K, Tanaka T, et al: Significant involvement of herpesvirus entry mediator in human esophageal squamous cell carcinoma. Cancer 120: 808-817, 2014.

14. Hokuto D, Sho M, Yamato I, Yasuda S, Obara S, Nomi T and Nakajima Y: Clinical impact of herpesvirus entry mediator expression in human hepatocellular carcinoma. Eur J Cancer 51: 157-165, 2015.

15. Han L, Wang W, Lu J, Kong F, Ma G, Zhu Y, Zhao D, Zhu J, Shuai W, Zhou Q, et al: AAV-sBTLA facilitates HSP70 vaccine-triggered prophylactic antitumor immunity against a murine melanoma pulmonary metastasis model in vivo. Cancer Lett 354: 398-406, 2014. 
16. Yi Y, He HW, Wang JX, Cai XY,Li YW, Zhou J, Cheng YF, Jin JJ, Fan J and Qiu SJ: The functional impairment of HCC-infiltrating $\gamma \delta \mathrm{T}$ cells, partially mediated by regulatory T cells in a TGF $\beta$ and IL-10-dependent manner. J Hepatol 58: 977-983, 2013.

17. Yi Y, Wu H, Gao Q, He HW, Li YW, Cai XY, Wang JX, Zhou J, Cheng YF, Jin JJ, et al: Interferon regulatory factor (IRF)-1 and IRF-2 are associated with prognosis and tumor invasion in HCC. Ann Surg Oncol 20: 267-276, 2013.

18. Livak KJ and Schmittgen TD: Analysis of relative gene expression data using real-time quantitative PCR and the 2(-Delta Delta C(T)) method. Methods 25: 402-408, 2001.

19. Li Y, Tang Y, Ye L, Liu B, Liu K, Chen J and Xue Q: Establishment of a hepatocellular carcinoma cell line with unique metastatic characteristics through in vivo selection and screening for metastasis-related genes through cDNA microarray. J Cancer Res Clin Oncol 129: 43-51, 2003.

20. Li Y, Tian B, Yang J, Zhao L, Wu X, Ye SL, Liu YK and Tang ZY: Stepwise metastatic human hepatocellular carcinoma cell model system with multiple metastatic potentials established through consecutive in vivo selection and studies on metastatic characteristics. J Cancer Res Clin Oncol 130: 460-468, 2004.

21. Song J, Ge Z, Yang X, Luo Q, Wang C, You H, Ge T, Deng Y, Lin H, Cui Y, et al: Hepatic stellate cells activated by acidic tumor microenvironment promote the metastasis of hepatocellular carcinoma via osteopontin. Cancer Lett 356: 713-720, 2015

22. Beldi G, Wu Y, Banz Y, Nowak M, Miller L, Enjyoji K, Haschemi A, Yegutkin GG, Candinas D, Exley $M$ and Robson MC: Natural killer T cell dysfunction in CD39-null mice protects against concanavalin A-induced hepatitis. Hepatology 48: 841-852, 2008.

23. Ren S, Tian Q, Amar N, Yu H, Rivard CJ, Caldwell C, Ng TL, Tu M, Liu Y, Gao D, et al: The immune checkpoint, HVEM may contribute to immune escape in non-small cell lung cancer lacking PD-L1 expression. Lung Cancer 125: 115-120, 2018.

24. Sine H, Marco D and Straten PT: Effector CD4 and CD8 $\mathrm{T}$ cells and their role in the tumor microenvironment. Cancer Microenviron 6: 123-133, 2013.

25. Marin-Acevedo JA, Dholaria B, Soyano AE, Knutson KL, Chumsri S and Lou Y: Next generation of immune checkpoint therapy in cancer: New developments and challenges. J Hematol Oncol 11: 39, 2018.

26. Zhao Q, Huang ZL, He M, Gao Z and Kuang DM: BTLA identifies dysfunctional PD-1-expressing $\mathrm{CD} 4^{+} \mathrm{T}$ cells in human hepatocellular carcinoma. Oncoimmunology 5: e1254855, 2016.

27. Cheng AL, Hsu C, Chan SL, Choo SP and Kudo M: Challenges of combination therapy with immune checkpoint inhibitors for hepatocellular carcinoma. J Hepatol 72: 307-319, 2020.

28. Darvin P, Toor SM, Sasidharan Nair V and Elkord E: Immune checkpoint inhibitors: Recent progress and potential biomarkers. Exp Mol Med 50: 1-11, 2018

29. Zhu AX, Finn RS, Edeline J, Cattan S, Ogasawara S, Palmer D, Verslype C, Zagonel V, Fartoux L, Vogel A, et al: Pembrolizumab in patients with advanced hepatocellular carcinoma previously treated with sorafenib (KEYNOTE-224): A non-randomised, open-label phase 2 trial. Lancet Oncol 19: 940-952, 2018
30. Fourcade J, Sun Z, Pagliano O, Guillaume P, Luescher IF, Sander C, Kirkwood JM, Olive D, Kuchroo V and Zarour HM: CD8(+) T cells specific for tumor antigens can be rendered dysfunctional by the tumor microenvironment through upregulation of the inhibitory receptors BTLA and PD-1. Cancer Res 72: 887-896, 2012.

31. Grabmeier-Pfistershammer K, Stecher C, Zettl M, Rosskopf S, Rieger A, Zlabinger GJ and Steinberger P: Antibodies targeting BTLA or TIM-3 enhance HIV-1 specific T cell responses in combination with PD-1 blockade. Clin Immunol 183: 167-173, 2017.

32. StecherC,BattinC,LeitnerJ,ZettlM,Grabmeier-PfistershammerK, Höller C,Zlabinger GJ and Steinberger P: PD-1 blockade promotes emerging checkpoint inhibitors in enhancing $\mathrm{T}$ cell responses to allogeneic dendritic cells. Front Immunol 8: 572, 2017.

33. Liu J, Li J, He M, Zhang GL and Zhao Q: Distinct changes of BTLA and HVEM expressions in circulating $\mathrm{CD}^{+}$and $\mathrm{CD}^{+} \mathrm{T}$ cells in hepatocellular carcinoma patients. $\mathrm{J}$ Immunol Res 2018: 4561571, 2018.

34. Inoue $T$, Sho $M$, Yasuda $S$, Nishiwada $S$, Nakamura $S$, Ueda $T$, Nishigori N, Kawasaki K, Obara S, Nakamoto T, et al: HVEM expression contributes to tumor progression and prognosis in human colorectal cancer. Anticancer Res 35: 1361-1367, 2015.

35. Lan X, Li S, Gao H, Nanding A, Quan L, Yang C, Ding S and Xue Y: Increased BTLA and HVEM in gastric cancer are associated with progression and poor prognosis. Onco Targets Ther 10: 919-926, 2017.

36. Tao R, Wang L, Murphy KM, Fraser CC and Hancock WW: Regulatory $\mathrm{T}$ cell expression of herpesvirus entry mediator suppresses the function of $\mathrm{B}$ and $\mathrm{T}$ lymphocyte attenuator-positive effector T cells. J Immunol 180: 6649-6655, 2008.

37. Derré L, Rivals JP, Jandus C, Pastor S, Rimoldi D, Romero P, Michielin O, Olive D and Speiser DE: BTLA mediates inhibition of human tumor-specific CD8+ T cells that can be partially reversed by vaccination. J Clin Invest 120: 157-167, 2010.

38. Haymaker CL, Wu RC, Ritthipichai K, Bernatchez C, Forget MA, Chen JQ, Liu H, Wang E, Marincola F, Hwu P and Radvanyi LG: BTLA marks a less-differentiated tumor-infiltrating lymphocyte subset in melanoma with enhanced survival properties. Oncoimmunology 4: e1014246, 2015.

39. Martins-Filho SN, Paiva C, Azevedo RS and Alves VAF: Histological grading of hepatocellular carcinoma-a systematic review of literature. Front Med (Lausanne) 4: 193, 2017.

40. Minagawa M, Ikai I, Matsuyama Y, Yamaoka Y and Makuuchi M: Staging of hepatocellular carcinoma: Assessment of the Japanese TNM and AJCC/UICC TNM systems in a cohort of 13,772 patients in Japan. Ann Surg 245: 909-922, 2007.

41. Forner A, Reig ME, de Lope CR and Bruix J: Current strategy for staging and treatment: The BCLC update and future prospects. Semin Liver Dis 30: 61-74, 2010. 